\title{
Response to Neoadjuvant Therapy and Prognosis in Patients with Resectable Pancreatic Cancer: A Propensity Score Matching Analysis
}

\author{
Min Sung Yoon ${ }^{1}$, Hee Seung Lee ${ }^{1}$, Chang Moo Kang ${ }^{2}$, Woo Jung Lee ${ }^{2}$, Jiyoung Keum¹, Min Je Sung ${ }^{1}$, Seung- \\ seob Kim ${ }^{3}$, Mi-Suk Park ${ }^{3}$, Jung Hyun Jo ${ }^{1}$, Moon Jae Chung ${ }^{1}$, Jeong Youp Park ${ }^{1}$, Seung Woo Park ${ }^{1}$, Si Young \\ Song ${ }^{1}$, Ho Kyoung Hwang ${ }^{2}$, and Seungmin Bang ${ }^{1}$ \\ ${ }^{1}$ Division of Gastroenterology, Department of Internal Medicine and ${ }^{2}$ Department of Hepatobiliary and Pancreatic Surgery, Yonsei \\ University College of Medicine, and ${ }^{3}$ Department of Radiology and Research Institute of Radiological Science, Severance Hospital, \\ Yonsei University College of Medicine, Seoul, Korea
}

See editorial on page 3 .

\author{
Article Info \\ Received October 6, 2020 \\ Revised March 12, 2021 \\ Accepted March 15, 2021 \\ Published online June 22, 2021 \\ Corresponding Author \\ Seungmin Bang \\ ORCID https://orcid.org/0000-0001-5209-8351 \\ E-mail bang7028@yuhs.ac

\section{Ho Kyoung Hwang} \\ ORCID https://orcid.org/0000-0003-4064-7776 \\ E-mail DRHHK@yuhs.ac
}

Min Sung Yoon and Hee Seung Lee contributed equally to this work as first authors.
Background/Aims: Controversy regarding the effectiveness of neoadjuvant therapy for resectable pancreatic ductal adenocarcinoma (PDAC) still exists. Here, we aimed to identify the potential benefits of neoadjuvant therapy followed by surgery for resectable PDAC.

Methods: We reviewed radiologically resectable PDAC patients who received resection with curative intent at a tertiary hospital in South Korea between January 2012 and August 2019. A total of 202 patients underwent curative resection for resectable PDAC: 167 underwent surgical resection first during this period, and 35 received neoadjuvant chemotherapy/chemoradiation therapy followed by surgery. Resectable PDAC patients were subdivided, and 1:3 propensity score matching (PSM) was performed to reduce selection bias.

Results: Compared with the group that received surgery first, the group that received neoadjuvant treatment followed by surgery had significantly smaller tumors $(22.0 \mathrm{~mm}$ vs $27.0 \mathrm{~mm}$, $p=0.004)$, a smaller proportion of patients with postoperative pathologic $T$ stage $(p=0.026)$, a smaller proportion of patients with lymphovascular invasion $(20.0 \%$ vs $40.7 \%, p=0.022)$, and a larger proportion of patients with negative resection margins $(74.3 \%$ vs $51.5 \%, p=0.049)$. After PSM, the group that received neoadjuvant therapy had a significantly longer progression-free survival than those in the group that underwent surgery first (29.6 months vs 15.1 months, $p=0.002$ ). Overall survival was not significantly different between the two groups after PSM analysis.

Conclusions: We observed significantly better surgical outcomes and progression-free survival with the addition of neoadjuvant therapy to the management of resectable PDAC. However, despite PSM, there was still selection bias due to the use of different regimens between the groups receiving surgery first and neoadjuvant therapy. Large homogeneous samples are needed in the future prospective studies. (Gut Liver 2022;16:118-128)

Key Words: Pancreatic neoplasm; Neoadjuvant therapy; Surgical outcome; Progression-free survival

\section{INTRODUCTION}

Surgical resection is the only curative treatment option in pancreatic ductal adenocarcinoma (PDAC). However, patients suitable for upfront surgery constitute less than $20 \%$ of all patients diagnosed with PDAC. Furthermore, $40 \%$ to $60 \%$ of patients experience tumor recurrence with- in the first 12 months after curative surgery. ${ }^{1-5}$ These results suggest two important clinical limitations of curative surgery for PDAC. First, the presence of micrometastases or microinvasion in the subset of resectable PDAC at the initial diagnosis cannot be detected with the currently available cross-sectional imaging (computed tomography [CT] scan and/or magnetic resonance imaging) in preoperative 
period. Second, the aggressive nature of surgery for PDAC, which is pylorus-preserving pancreaticoduodenectomy, prevents patients from receiving adjuvant chemotherapy with surgery-related morbidity and complications. ${ }^{6,7}$ According to previous studies, only $26 \%$ to $74 \%$ of patients receive postoperative therapy because of postoperative complications, reduced performance status, and early disease recurrence. ${ }^{8-11}$

Neoadjuvant therapy for PDAC has been considered an alternative option in borderline resectable/locally advanced PDAC. Neoadjuvant chemotherapy has more potential benefits than the traditional upfront surgery approach, including the following: it eradicates micrometastases, increases negative margin resection rates, and avoids futile surgery by identifying rapidly progressive tumor types. ${ }^{12-15}$ Furthermore, neoadjuvant therapy also likely promotes better patient selection by excluding patients with undetected distant disease or rapidly progressive disease who will not benefit from resection and for whom the associated morbidity of surgical resection is avoided. ${ }^{16,17}$

However, controversy regarding the effectiveness of neoadjuvant therapy in resectable PDAC still exists. Here, we retrospectively analyzed a single-center pancreatic cancer database to identify the potential benefits of neoadjuvant therapy.

\section{MATERIALS AND METHODS}

\section{Study population}

This was a single-center retrospective study comprising patients who underwent curative-intent pancreatectomy for resectable PDAC. We identified all patients who received curative-intent resection for pancreatic cancer at Severance Hospital between January 2012 and August 2019 by searching the electronic medical records. A total of 284 patients underwent surgical resection for pancreatic cancer during this period (Fig. 1). The exclusion criteria were as follows: (1) pancreatic tumors except PDAC $(\mathrm{n}=31)$ and (2) borderline resectable $(n=38)$, locally advanced $(n=11)$, and metastatic $(n=2)$ PDAC.

As a result, 202 patients were enrolled in this study and divided into two groups: those undergoing upfront surgery $(\mathrm{n}=167,82.7 \%)$ and those receiving neoadjuvant therapy

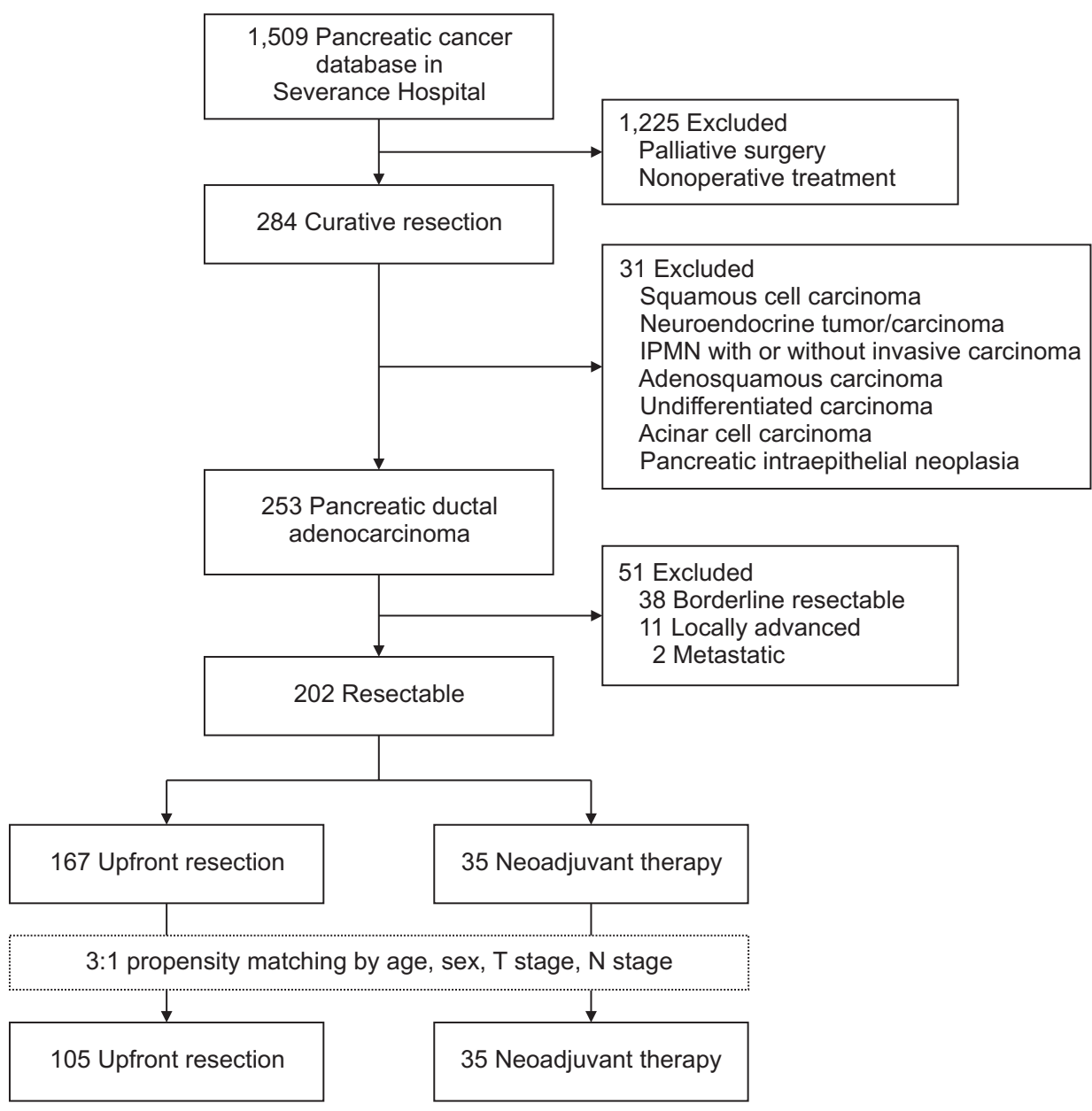

Fig. 1. Patient diagram. IPMN, intraductal papillary mucinous neoplasm. 
followed by surgery ( $\mathrm{n}=35,17.3 \%)$. To reduce survival bias according to chemotherapy itself in neoadjuvant therapy group compared with upfront surgery group, we also excluded patients who underwent upfront surgery alone without adjuvant chemotherapy $(\mathrm{n}=28)$ in subgroup analysis.

The surgical procedures that patients underwent were as follows: pylorus-preserving pancreaticoduodenectomy $(\mathrm{n}=101)$, Whipple's operation $(\mathrm{n}=4)$, distal pancreatectomy $(\mathrm{n}=88)$, and total pancreatectomy $(\mathrm{n}=9)$. Regarding chemotherapeutic regimen, adjuvant and neoadjuvant chemotherapies mostly comprised gemcitabine ( $n=123 / 158$, $77.8 \%$ ) and 5-fluorouracil (5-FU), leucovorin, irinotecan, and oxaliplatin (FOLFIRINOX) ( $\mathrm{n}=17 / 20,85 \%)$, respectively. This study was performed in accordance with ethical guidelines of the 1975 Declaration of Helsinki and approved by the Institutional Review Boards of Severance Hospital (IRB number: 4-2015-1058). Given its retrospective nature, written informed consent was not required to access clinical data.

\section{Preoperative evaluation}

Patients underwent preoperative evaluation, including clinical history assessment, physical examination, laboratory assessment, and tumor marker and preoperative radiologic tests with three-phase pancreas CT scan, abdominal magnetic resonance imaging, and positron emission tomography-CT. Endoscopic ultrasound-guided fine needle aspiration was also performed for the pathologic confirmation of PDAC before the administration of neoadjuvant therapy. Tumor resectability was confirmed based on the three-phase pancreas CT scan and abdominal magnetic resonance imaging according to the following National Comprehensive Cancer Network guidelines: no tumor contact with the superior mesenteric vein or portal vein or $\leq 180^{\circ}$ contact without vein contour irregularity and no contact with the celiac axis, superior mesenteric artery, or common hepatic artery. All preoperative radiologic images were reviewed by two abdominal radiologists who were blinded to the surgical and pathologic results. They noted the presence of metastatic lymph nodes, which were defined as those having a short axis longer than $1 \mathrm{~cm}$, a round contour, heterogeneity, or central necrosis.

\section{Variables}

Patients were recorded as having hypertension, diabetes mellitus, chronic pancreatitis, smoking history, alcohol history, and family history of pancreatic cancer if they had these factors prior to the pancreatic cancer diagnosis date. We recorded the first serum carbohydrate antigen 19-9 level $(\mathrm{U} / \mathrm{mL})$, which was obtained before patients under- went surgery or received neoadjuvant chemotherapy.

Upfront surgery was defined as neither preoperative chemotherapy nor concurrent chemoradiotherapy (CCRT) administration, regardless if adjuvant therapy was followed. Neoadjuvant therapy was defined as the administration of chemotherapy with or without concurrent radiation before surgery (CCRT and chemotherapy).

We retrieved the following data from the preoperative imaging test: tumor location (head/body/tail), arterial involvement (celiac artery/superior mesenteric artery/ common hepatic artery), venous involvement (portal vein/ superior mesenteric vein), radiologic tumor size, regional lymph node involvement, distant metastasis, pancreatic duct dilatation, and bile duct dilatation. Clinical and pathologic $\mathrm{T}, \mathrm{N}$, and $\mathrm{M}$ staging were defined according to the 8th edition of the American Joint Committee on Cancer staging system.

Additionally, the following pathological report was investigated: pathologic tumor size at its largest diameter, lymphovascular invasion, perineural invasion, pathologic differentiation, resection margin status, and number of resected and involved lymph nodes. Resection margin status was classified as negative (R0), microscopically positive or tumor-free margin $<1 \mathrm{~mm}(\mathrm{R} 1)$, and macroscopically positive (R2). Overall survival (OS) and progression-free survival (PFS) were measured from the date of diagnosis until either death from any cause or detection of first recurrence. Tumor recurrence was divided into local recurrence and distant recurrence.

\section{Statistical analyses}

Baseline characteristics were summarized as the mean (standard deviation) or the median (interquartile range, IQR) for continuous variables and as numbers (percentage) for categorical variables. Tests of differences were performed using the Student $t$-test or the Mann-Whitney $\mathrm{U}$ test for continuous variables and the Fisher exact test for categorical variables. Survival curves were estimated using the Kaplan-Meier method, and the log-rank test was used to compare survivals between the two groups. To reduce immortal bias we also performed time-dependent covariant analysis. A 1:3 propensity score matching (PSM) was performed to reduce selection bias between the allocation of the neoadjuvant and upfront surgery groups. Propensity score models were adjusted for age, sex, preoperative $\mathrm{T}$ stage, and preoperative $\mathrm{N}$ stage. Nearest-neighbor matching was used. p-value $<0.05$ was considered statistically significant. PSM was performed using the MatchIt package in R. All statistical analyses were performed using R software version 3.6.1 (The R Foundation, Vienna, Austria). 


\section{RESULTS}

\section{Patient characteristics}

Table 1 presents the baseline characteristics of 167 patients who underwent upfront surgery and 35 who received neoadjuvant therapy for resectable PDAC. Using unmatched data, age and clinical node stage showed differences between the two groups. To reduce selection bias, we performed 1:3 PSM based on age, sex, and clinical $\mathrm{T}$ and $\mathrm{N}$ stages for comparable analysis. After PSM, 35 patients in the neoadjuvant therapy group were matched with 105 patients who underwent upfront surgery (Fig. 1). Both groups were balanced for preoperative baseline characteristics.

Thirty-five patients received neoadjuvant treatment. Of these patients, $57 \%(\mathrm{n}=20)$ received neoadjuvant chemotherapy without radiation therapy and $43 \%(n=15)$ received CCRT. The neoadjuvant chemotherapy group received a median of four cycles (IQR, four to five cycles), mainly with FOLFIRINOX ( $n=17)$. Other chemotherapeutic agents administered included gemcitabine/nab-paclitaxel $(\mathrm{n}=1)$, gemcitabine/erlotinib $(\mathrm{n}=1)$, and gemcitabine/ cisplatin $(n=1)$. The neoadjuvant chemoradiation group received various chemotherapeutic agents, including 5-FU/ leucovorin $(\mathrm{n}=3)$, gemcitabine $(\mathrm{n}=4)$, and oral $5-\mathrm{FU}(\mathrm{n}=8)$. Regarding the neoadjuvant therapy group, $77.1 \%(n=27 / 35)$ of patients also had a median of two cycles (IQR, two to three cycles) of adjuvant chemotherapy with gemcitabine $(\mathrm{n}=19)$, gemcitabine/capecitabine $(\mathrm{n}=4)$, gemcitabine/erlotinib ( $\mathrm{n}=2), 5-\mathrm{FU} /$ epirubicin/cisplatin $(\mathrm{n}=1)$, and 5-FU/cisplatin $(n=1)$. In the upfront surgery group, $81.4 \%(n=136)$ of patients received adjuvant therapy followed by surgery with a median of six cycles. Moreover, 96.3\% ( $n=131 / 136)$ of patients received adjuvant chemotherapy without radiation therapy, and the remaining $3.7 \%(n=5 / 136)$ of patients received CCRT. Gemcitabine ( $\mathrm{n}=104)$ was the most commonly used regimen in adjuvant chemotherapy, followed by 5 -FU, etoposide, and cisplatin $(\mathrm{n}=8)$, gemcitabine/ capecitabine $(\mathrm{n}=8), 5-\mathrm{FU} /$ cisplatin $(\mathrm{n}=6), 5$-FU/leucovorin $(n=2)$, tegafur/uracil $(n=2)$, and FOLFIRINOX $(n=1)$.

\section{Surgical outcomes}

We found some variants that showed more statistically significant improvement in the neoadjuvant therapy group than in the upfront surgery group (Table 2). The $\mathrm{R} 0$ resection rate was significantly higher in the neoadjuvant therapy group than that in the upfront surgery group (74.3\% vs $51.5 \%, \mathrm{p}=0.049)$. Pathologic tumor size $(22.0$ $\mathrm{mm}$ vs $27.0 \mathrm{~mm}, \mathrm{p}=0.004)$ and pathologic $\mathrm{T}$ stage $(\mathrm{p}=0.026)$ were significantly lower in the neoadjuvant therapy group than those in the upfront surgery group. Patients receiving neoadjuvant therapy also had significant improvement in lymphovascular invasion ( $20.0 \%$ vs $40.7 \%, \mathrm{p}=0.022$ ). Positive lymph node/sampled lymph node ratio was lower in the neoadjuvant therapy group than that in the upfront surgery group. However, the difference was not statistically significant between the two groups ( $5 \%$ vs $9 \%, \mathrm{p}=0.115$ ).

After 1:3 PSM, statistically significant improvement of pathologic tumor size $(22.0 \mathrm{~mm}$ vs $28.0 \mathrm{~mm}, \mathrm{p}=0.001)$, pathologic T stage $(\mathrm{p}=0.009)$, degree of histologic differentiation ( $\mathrm{p}=0.025)$, lymphovascular invasion $(20.0 \%$ vs $52.4 \%, \mathrm{p}=0.001)$, and $\mathrm{R} 0$ resection margin rates $(74.3 \%$ vs $49.5 \%, \mathrm{p}=0.034)$ were also shown in the matched groups. Furthermore, the difference between the number of positive lymph node ( 0.74 vs $1.66, \mathrm{p}=0.025$ ), positive lymph node/sampled lymph node ratio ( $5 \%$ vs $11 \%, \mathrm{p}=0.038$ ), and pathologic $\mathrm{N}$ stage ( $\mathrm{p}=0.031$ ) were found to be statistically significant between the two groups, which was not detected in raw data. On pathologic review in patients who received neoadjuvant treatment, we checked their College of American Pathologists score (modified Ryan scheme for tumor regression score) in four patients (score 2 in three patients and score 0 in one patient). Patient with score 0 showed no viable cancer cells in their surgical specimens.

\section{Prognostic outcome}

The median follow-up durations were 23.0 months (range, 15.5 to 37.2 months) and 19.3 months (range, 6.7 to 29.6 months) for patients in the upfront surgery and neoadjuvant therapy groups, respectively. During the followup period, $71.9 \%(120 / 167)$ and $37.1 \%$ (13/35) of patients in the upfront surgery group and the neoadjuvant therapy group exhibited recurrence, respectively. Compared with upfront surgery, neoadjuvant therapy had a more significant improvement in PFS in both the unmatched $(\mathrm{p}=0.007)$ and matched groups ( $\mathrm{p}=0.002$ ) (Fig. 2). The median PFS in the matched groups were 29.6 and 15.1 months for patients in the neoadjuvant therapy and upfront surgery groups, respectively. Among upfront surgery group, patients who received adjuvant chemotherapy following upfront surgery were compared with the neoadjuvant therapy group. The neoadjuvant group had a more significant improvement in PFS in both the matched $(\mathrm{p}=0.023)$ and unmatched groups $(\mathrm{p}=0.042)$ (Supplementary Fig. 1). OS was not significantly different between the two groups ( $\mathrm{p}=0.310)$, even after PSM analysis $(\mathrm{p}=0.290)$ because of a short follow-up duration (Fig. 3). We also analyzed after excluding the neoadjuvant therapy alone without adjuvant chemotherapy $(n=8)$. Compared with upfront surgery, neoadjuvant therapy showed a trend towards prolonged PFS in matched groups $(\mathrm{p}=0.055)$, but not in OS $(\mathrm{p}=0.81)$.

Probability of survival may be overestimated in this 


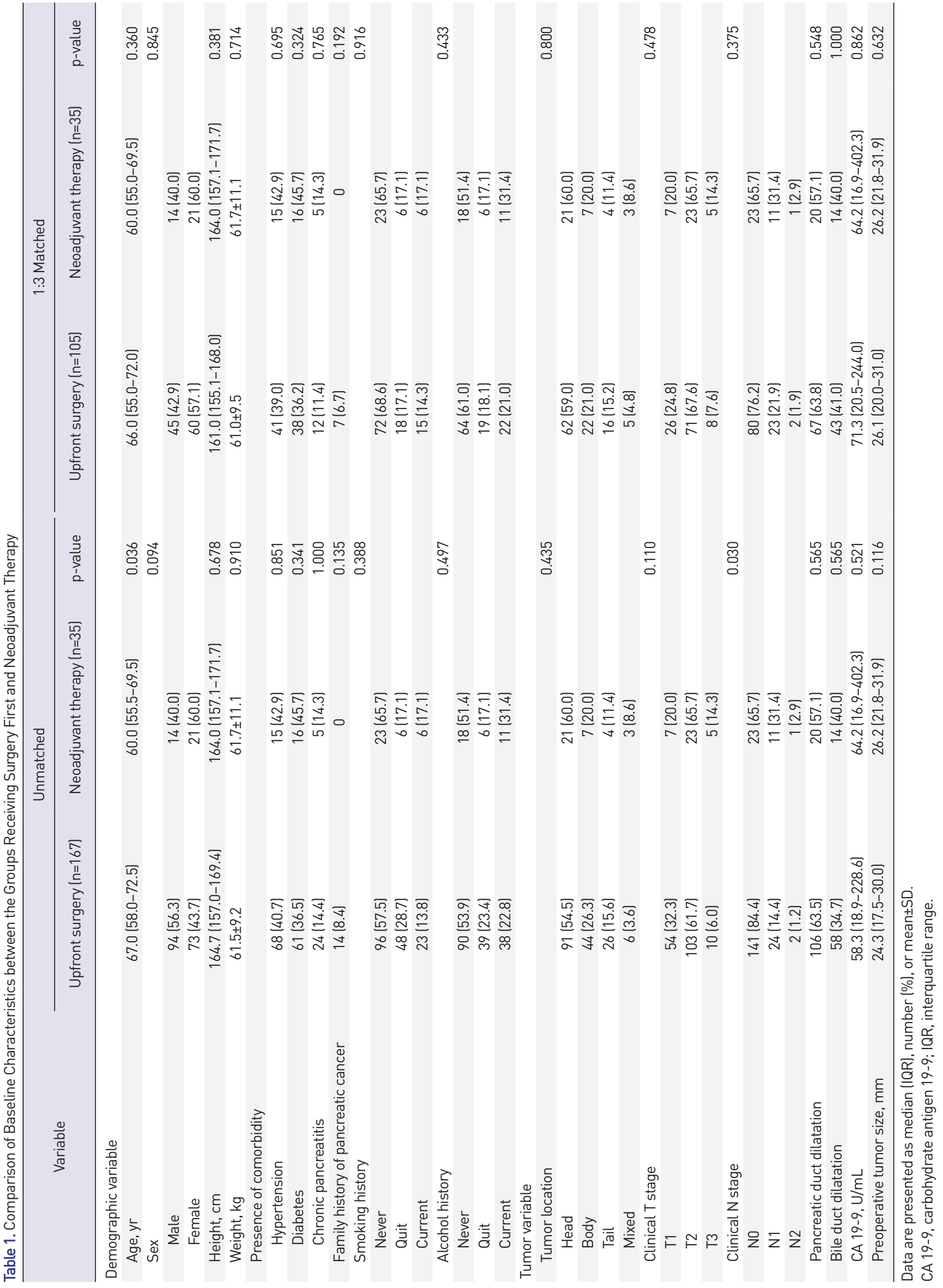




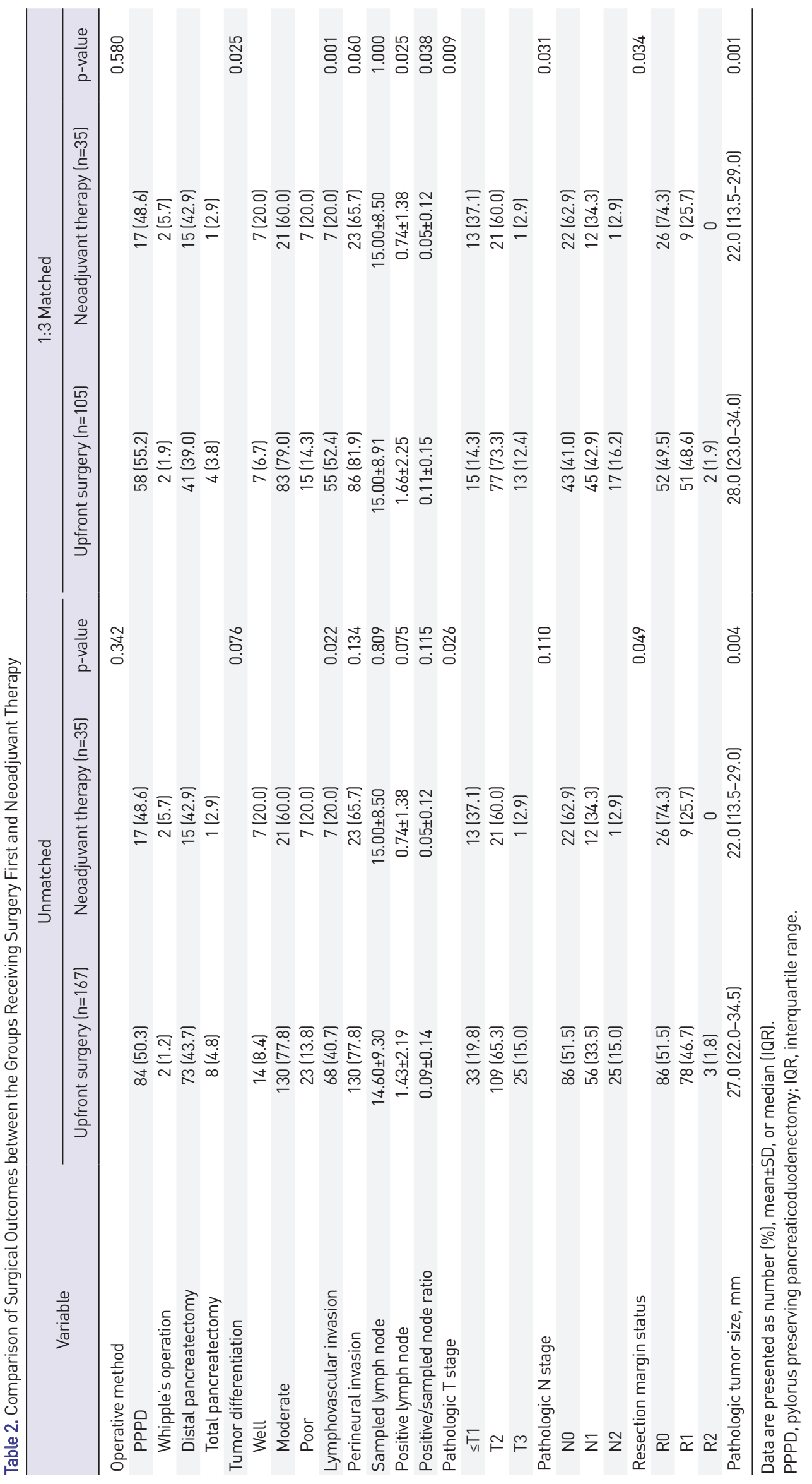



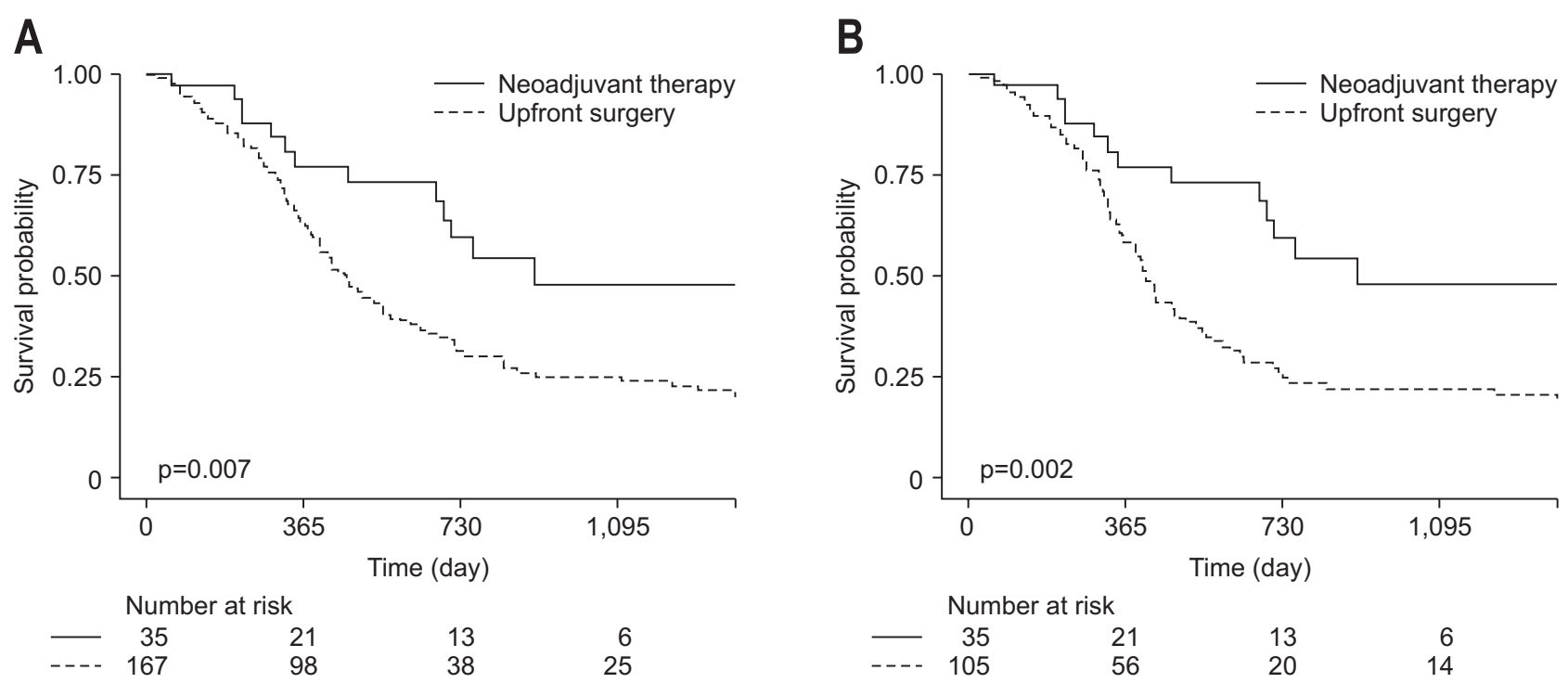

Fig. 2. Kaplan-Meier plot of progression-free survival comparing the neoadjuvant therapy group and the group receiving surgery first. (A) Unmatched and (B) matched data are presented.
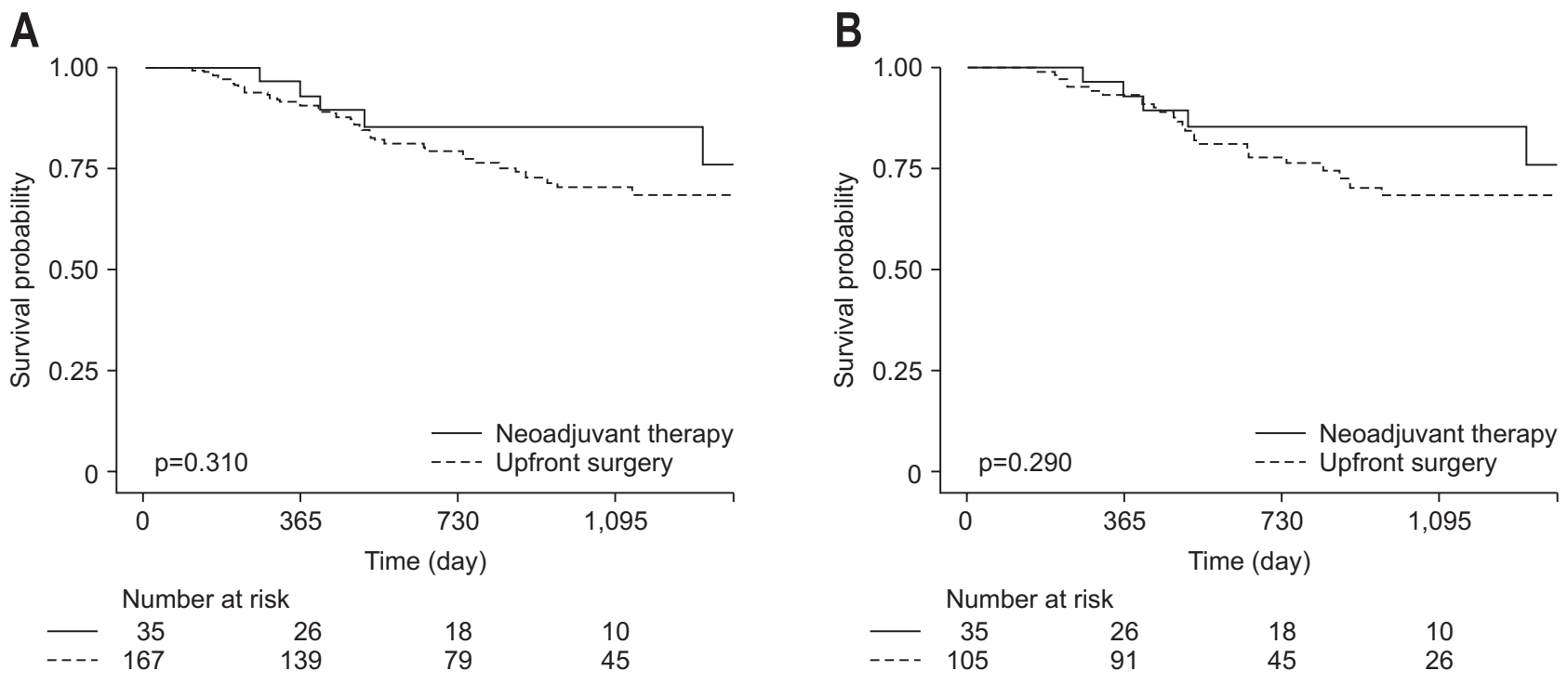

Fig. 3. Kaplan-Meier plot of overall survival comparing the neoadjuvant therapy group and the group receiving surgery first. (A) Unmatched and (B) matched data are presented.

study because some patients were loss to follow-up and the number of samples is small. Time-dependent Cox regression analysis did not show the difference in OS between upfront surgery and neoadjuvant therapy (unmatched $\mathrm{p}=0.4$; hazard ratio, $0.7 ; 95 \%$ confidence interval [CI], 0.29 to 1.65 and matched $\mathrm{p}=0.5$; hazard ratio, $0.75 ; 95 \% \mathrm{CI}, 0.30$ to 1.83 ). After all, neoadjuvant therapy showed statistically prolonged PFS even in time-dependent analysis $(\mathrm{p}=0.017)$ (Supplementary Table 1). On univariate analysis, older age, alcohol history, and neoadjuvant therapy were significant prognostic factors associated with PFS. After adjusting confounding factors, neoadjuvant therapy was statistically significant, independent of the better prognostic factor (hazard ratio, 0.39; $\mathrm{p}=0.002$ ) (Fig. 4).

\section{DISCUSSION}

In this study, we found that postoperative pathologic finding and PFS were significantly better in the neoadjuvant therapy group than those in the upfront surgery group. The theoretical advantages of neoadjuvant therapy include the following: early treatment of micrometastases, sparing patients who already have occult metastases, 


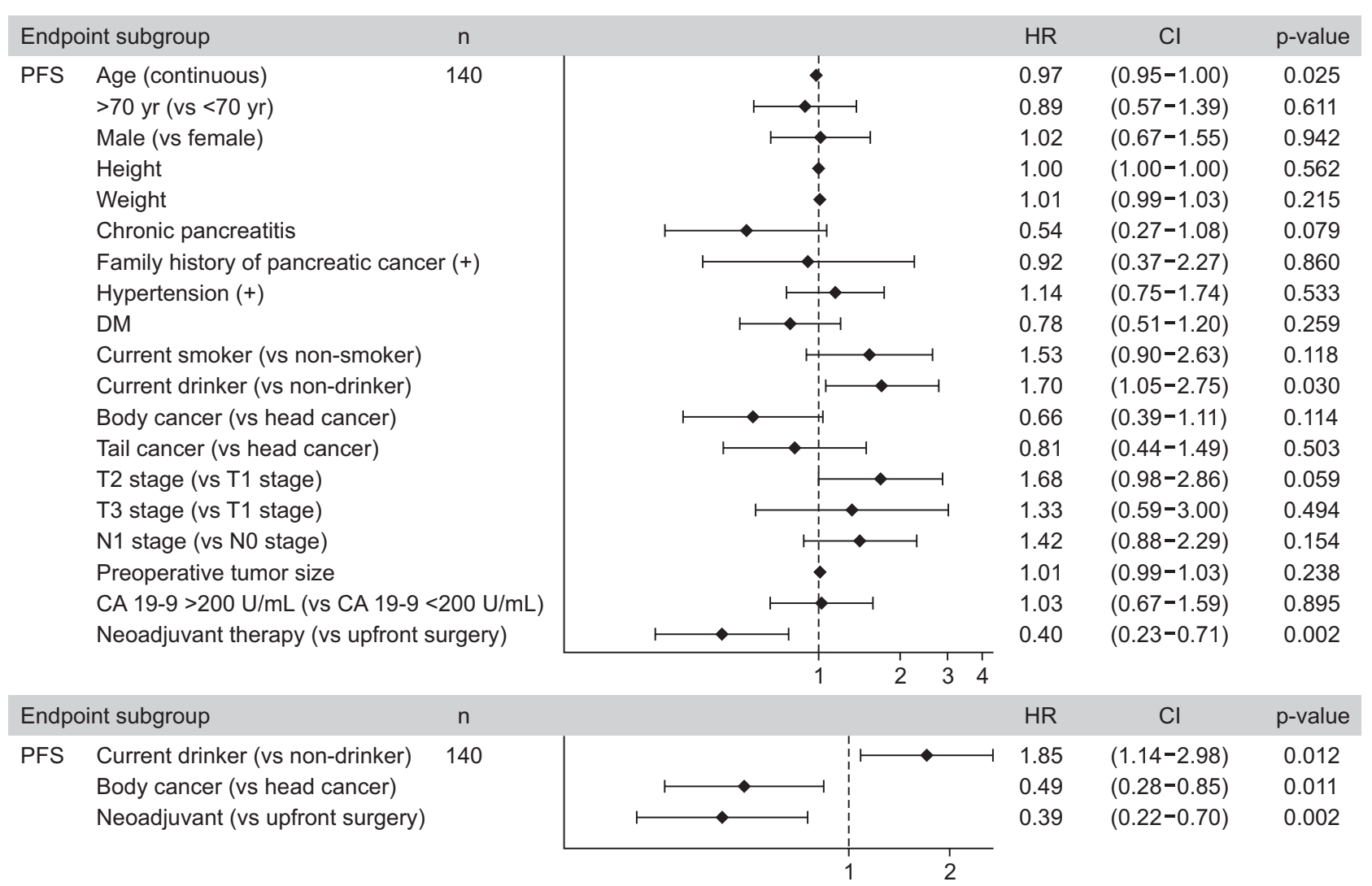

Fig. 4. Multivariate Cox regression analysis of progression-free survival (PFS).

DM, diabetes mellitus; CA 19-9, carbohydrate antigen 19-9; HR, hazard ratio; $\mathrm{Cl}$, confidence interval.

reduced risk of tumor seeding at the time of surgery, and improved tolerance compared with upfront surgery.

A few studies showed that neoadjuvant therapy has potential advantages over upfront surgery in resectable PDAC. ${ }^{13-15}$ Mokdad et al. ${ }^{14}$ reported that the neoadjuvant therapy group was associated with more improved survival than the upfront surgery group (median OS, 26 months vs 21 months; $\mathrm{p}<0.01)$. Ren et al..$^{15}$ reviewed 11 studies and reported that neoadjuvant therapy had higher R0 resection rate (odds ratio, $1.89 ; 95 \% \mathrm{CI}, 1.26$ to 2.83 ) and lower positive lymph node rate (odds ratio, $0.34 ; 95 \% \mathrm{CI}, 0.31$ to 0.37 ) than upfront surgery. However, previous studies used clinical stage I and II disease as proxies for resectable disease as there is no direct way to assess resectability in their cancer registry. ${ }^{14,18,19}$ Therefore, by definition, borderline resectable PDAC patients might have been enrolled in those studies. In real-world clinical practice, resectability is more important than tumor stage in determining the resection in pancreatic cancer. Therefore, strict measurement of resectability should be performed to evaluate the effect of neoadjuvant therapy for resectable PDAC. In the present study, we utilized the clinicoradiologic database. Specialized radiologists reviewed all images according to resectability based on the National Comprehensive Cancer
Network guidelines. ${ }^{20}$ Several previous studies also did not provide sufficient information on the type of chemotherapeutic agents used in their studies. Although the data set includes information on the receipt of adjuvant therapy, whether the intended postoperative therapy was completed is unknown. However, our study investigated all clinical information and chemotherapeutic regimens related to pancreatic cancer resection.

A recent first prospective randomized clinical trial on neoadjuvant chemoradiotherapy has reported that the R0 resection rate and pathologic parameters were superior with preoperative chemoradiotherapy, but it did not demonstrate an OS benefit. ${ }^{21}$ Although neoadjuvant therapy appears to have a more significant role in improving R0 resection rates in patients with borderline resectable pancreatic cancer (BRPC) and locally advanced pancreatic cancer (LAPC) compared with upfront resection, a statistically significant survival benefit has not routinely been demonstrated in the resectable cohort. ${ }^{22-24}$ Similarly, the benefits of neoadjuvant therapy on OS in resectable PDAC are different between studies. In the present study, OS was not different in the neoadjuvant therapy and upfront surgery groups. These effects may be attributed to several possible mechanisms. First, the prognosis of PDAC is poor irre- 
spective of minimal therapy change. A minor effect of neoadjuvant therapy has no effect on OS. Above all, surgery is still the first treatment of choice in resectable PDAC, and the possibility of micrometastasis is significantly higher in BRPC or LAPC than in resectable PDAC. Therefore, neoadjuvant therapy showed significantly prolonged OS in BRPC and LAPC in previous studies. ${ }^{10,25}$ On the contrary, a previous study showed that patients with metastatic lymph nodes experience OS benefits in a subgroup analysis. Neoadjuvant chemotherapy with gemcitabine-based regimens improved the prognosis of node-positive resectable PDAC.

Neoadjuvant FOLFIRINOX for BRPC or LAPC is feasible, with high R0 resection rates and prolonged median PFS and OS. However, a study evaluating the outcome for resectable PDAC has not been conducted yet. Except for ongoing clinical trials, our study is the first retrospective study to investigate the potential benefits of neoadjuvant therapy including FOLFIRINOX chemotherapy for resectable PDAC. In a subgroup analysis, our study did not confirm the benefits of neoadjuvant FOLFIRINOX on resectable PDAC because the recurrence event did not reach the median values due to short-term follow-up duration (data not shown). Neoadjuvant FOLFIRINOX might further improve the outcome and is currently being investigated in the PANACHE01-PRODIGE48 trial (NCT02959879), NorPACT-1 trial (NCT02919787), and PREOPANC2 trial (Netherlands Trial, NTR7292).

Regarding neoadjuvant therapy, besides not experiencing the benefits of this therapy, some patients may also be diagnosed with unresectable diseases after receiving this type of therapy. Failure rate has been reported to be as high as $16 \%{ }^{26}$ However, it is presumed that these patients experience an early recurrence even if they had undergone early surgery. Results from the recent two studies were similar: $70 \%$ to $75 \%$ of patients assigned to upfront surgery compared with $57.5 \%$ to $61 \%$ of patients assigned to neoadjuvant arm undergoing resection. ${ }^{22,27}$ The $\mathrm{R} 0$ resection rate was usually higher in neoadjuvant therapy than that in upfront surgery. Therefore, successful surgical resection rate was higher in the neoadjuvant therapy group than that in the upfront surgery group. The resection rate observed in a recent prospective clinical trial for resectable PDAC was consistent with that in a previous study. Therefore, besides increasing the rate of free margin resection and reducing the risk of local recurrence, neoadjuvant treatment allows the identification of rapidly metastatic patients and thus prevents them from undergoing a nonprofitable morbid surgery.

However, determining the characteristics of patients who benefit from neoadjuvant therapy is considered im- portant. Even if we consider neoadjuvant therapy because even margin-negative pancreatectomy followed by adjuvant chemotherapy can lead to high rates of tumor recurrence and poor survival outcomes, neoadjuvant therapy for resectable PDAC might be considered an overtreatment, subsequently resulting in unnecessary complications in some patients. The National Comprehensive Cancer Network guidelines recommend neoadjuvant therapy for resectable patients with high-risk features such as highly elevated carbohydrate antigen 19-9, large tumor size, large regional lymph node, excessive weight loss, and extreme pain. ${ }^{28,29}$ Recently, circulating tumor DNA and circulating tumor cells have been considered predictive markers related to preoperative and postoperative outcome and prognosis. ${ }^{30,31}$ Assessing circulating tumor DNA and circulating tumor cells at diagnosis is considered beneficial for patient selection. Not only conventional markers such as carbohydrate antigen 19-9 and tumor size but also circulating tumor DNA and circulating tumor cells might be predictive markers during patient selection. Furthermore, biologic behavior is more important than cancer tissue extent when assessing susceptibility to neoadjuvant therapy. Therefore, classifying PDAC tissue is considered significantly appropriate. For example, PDAC patients with homologous recombination deficiency should appropriately receive FOLFIRINOX or platinum-based chemotherapy. The risk for disease progression and losing a curative surgical window highlights the need for appropriate patient identification, further discovery of superior biomarkers or molecular profiles related to positive treatment response, and additional prospective comparative studies.

The present study has several limitations. First, this is an observational retrospective study with a small number of patients (Supplementary Tables 2, 3). In an effort to reduce selection bias, we performed PSM only for clinical factors that showed differences between two groups. Due to the restriction of retrospective analysis, it was impossible to define strict enrollment criteria of neoadjuvant group. However, we found patients in this study usually received neoadjuvant therapy in case of high $\mathrm{N}$ stage and large primary tumor size. Second, only patients with resectable PDAC who underwent tumor resection and/or received adjuvant or neoadjuvant treatment were enrolled. Patients in the neoadjuvant therapy group represent only those patients who tolerated neoadjuvant therapy and underwent resection which might result in biased estimates. With this study, we were unable to identify all patients who received chemotherapy with intent for later resection but who did not proceed with resection. However, in fact, these patients could have been potentially unresectable, even at the time of upfront surgery. Rather, since this study 
uses a nonrandomized retrospective study design, there is a high probability that patients who are expected to experience less benefits from the upfront surgery received neoadjuvant therapy. Nevertheless, surgical outcomes are more favorable in patients in the neoadjuvant therapy group than those in patients in the upfront surgery group. A well-randomized, prospective study is needed to examine this further. Third, neoadjuvant therapy comprises various chemotherapeutic and chemoradiotherapeutic regimens, with FOLFIRINOX being the most commonly used chemotherapeutic regimen. This can still cause selection bias despite of PSM in this study. We selected patients who received adjuvant gemcitabine-based therapy (upfront surgery group, $\mathrm{n}=112$ ) and neoadjuvant gemcitabinebased therapy (neoadjuvant therapy, $n=7$ ). After PSM, PFS was still statistically significantly prolonged in neoadjuvant therapy group compared with upfront surgery group $(\mathrm{p}=0.034)$. Nonetheless, selection bias is still an issue in the present study. Comprising large homogeneous samples are required in the future prospective studies.

In conclusion, we demonstrated the statistically significant improvement of surgical outcomes and PFS with neoadjuvant therapy in resectable PDAC. Despite these favorable findings, there was no difference in the OS between the two groups. Therefore, considering its association with long-term survival, better predictors of response and more effective preoperative regimens should be studied.

\section{CONFLICTS OF INTEREST}

No potential conflict of interest relevant to this article was reported.

\section{ACKNOWLEDGEMENTS}

This study was supported by a grant of the Korea Health Technology R\&D Project through the Korea Health Industry Development Institute, funded by the Ministry of Health and Welfare, Republic of Korea (grant number: HI19C0642-060019).

\section{AUTHOR CONTRIBUTIONS}

Study concept and design: M.S.Y., H.S.L. Data acquisition: M.S.Y., H.S.L., J.K., M.J.S. Data analysis and interpretation: M.S.Y., H.S.L. Drafting of the manuscript: M.S.Y., H.S.L. Critical revision of the manuscript: M.S.Y., H.S.L., C.M.K., W.J.L., J.K., M.J.S., S.S.K., M.S.P., J.H.J., M.J.C.,
J.Y.P., S.W.P., S.Y.S. Study supervision: H.K.H., S.B. All authors read and approved the final manuscript.

\section{ORCID}

Min Sung Yoon https://orcid.org/0000-0002-4513-4948 Hee Seung Lee https://orcid.org/0000-0002-2825-3160 Chang Moo Kang https://orcid.org/0000-0002-5382-4658 Woo Jung Lee https://orcid.org/0000-0001-9273-261X Jiyoung Keum https://orcid.org/0000-0001-7061-6183 Min Je Sung https://orcid.org/0000-0001-5395-8851 Seung-seob Kim https://orcid.org/0000-0001-6071-306X Mi-Suk Park https://orcid.org/0000-0001-5817-2444 Jung Hyun Jo https://orcid.org/0000-0002-2641-8873 Moon Jae Chung https://orcid.org/0000-0002-5920- 8549 Jeong Youp Park https://orcid.org/0000-0003-0110-8606 Seung Woo Park https://orcid.org/0000-0001-8230-964X Si Young Song https://orcid.org/0000-0002-1417-4314 Ho Kyoung Hwang https://orcid.org/0000-0003-4064-7776 Seungmin Bang https://orcid.org/0000-0001-5209-8351

\section{SUPPLEMENTARY MATERIALS}

Supplementary materials can be accessed at https://doi. org/10.5009/gnl20301.

\section{REFERENCES}

1. Miller KD, Siegel RL, Lin CC, et al. Cancer treatment and survivorship statistics, 2016. CA Cancer J Clin 2016;66:271289.

2. Surveillance, Epidemiology, and End Results (SEER) Program. Cancer stat facts: pancreas cancer [Internet]. Bethesda: National Cancer Institute [cited 2016 Jul 13]. Available from: http://seer.cancer.gov/statfacts/html/pancreas.html.

3. Oettle H, Neuhaus P, Hochhaus A, et al. Adjuvant chemotherapy with gemcitabine and long-term outcomes among patients with resected pancreatic cancer: the CONKO-001 randomized trial. JAMA 2013;310:1473-1481.

4. Kurahara H, Maemura K, Mataki Y, et al. A therapeutic strategy for resectable pancreatic cancer based on risk factors of early recurrence. Pancreas 2018;47:753-758.

5. Nishio K, Kimura K, Amano R, et al. Preoperative predictors for early recurrence of resectable pancreatic cancer. World J Surg Oncol 2017;15:16.

6. Schwarz L, Vernerey D, Bachet JB, et al. Resectable pancreatic adenocarcinoma neo-adjuvant FOLF(IRIN)OX-based chemotherapy: a multicenter, non-comparative, random- 
ized, phase II trial (PANACHE01-PRODIGE48 study). BMC Cancer 2018;18:762.

7. Lee HS, An C, Hwang HK, et al. Preoperative prediction of futile surgery in patients with radiologically resectable or borderline resectable pancreatic adenocarcinoma. J Gastroenterol Hepatol 2020;35:499-507.

8. Tzeng CW, Fleming JB, Lee JE, et al. Defined clinical classifications are associated with outcome of patients with anatomically resectable pancreatic adenocarcinoma treated with neoadjuvant therapy. Ann Surg Oncol 2012;19:2045-2053.

9. Tajima H, Ohta T, Okazaki M, et al. Neoadjuvant chemotherapy with gemcitabine-based regimens improves the prognosis of node positive resectable pancreatic head cancer. Mol Clin Oncol 2019;11:157-166.

10. Petrelli F, Coinu A, Borgonovo K, et al. FOLFIRINOX-based neoadjuvant therapy in borderline resectable or unresectable pancreatic cancer: a meta-analytical review of published studies. Pancreas 2015;44:515-521.

11. Bakens MJ, van der Geest LG, van Putten M, et al. The use of adjuvant chemotherapy for pancreatic cancer varies widely between hospitals: a nationwide population-based analysis. Cancer Med 2016;5:2825-2831.

12. Bradley A, Van Der Meer R. Upfront surgery versus neoadjuvant therapy for resectable pancreatic cancer: systematic review and Bayesian network meta-analysis. Sci Rep 2019;9:4354.

13. Motoi F, Kosuge T, Ueno H, et al. Randomized phase II/III trial of neoadjuvant chemotherapy with gemcitabine and S-1 versus upfront surgery for resectable pancreatic cancer (Prep-02/JSAP05). Jpn J Clin Oncol 2019;49:190-194.

14. Mokdad AA, Minter RM, Zhu H, et al. Neoadjuvant therapy followed by resection versus upfront resection for resectable pancreatic cancer: a propensity score matched analysis. J Clin Oncol 2017;35:515-522.

15. Ren X, Wei X, Ding Y, et al. Comparison of neoadjuvant therapy and upfront surgery in resectable pancreatic cancer: a meta-analysis and systematic review. Onco Targets Ther 2019;12:733-744.

16. Raufi AG, Manji GA, Chabot JA, Bates SE. Neoadjuvant treatment for pancreatic cancer. Semin Oncol 2019;46:19-27.

17. Blair AB, Sorber R, Rozich NS, Burkhart RA. A qualitative review of neoadjuvant chemotherapy in resectable pancreatic adenocarcinoma. Pancreas 2019;48:973-984.

18. Datta SK, Belini G, Singh M, et al. Survival outcomes between surgery with adjuvant therapy compared to neoadjuvant therapy with surgery in stage I pancreatic adenocarcinoma: results from a large national cancer database. J Clin Oncol 2019;37(4 Suppl):335-335.

19. Hashmi A, Kozick Z, Fluck M, et al. Neoadjuvant versus adjuvant chemotherapy for resectable pancreatic adeno- carcinoma: a national cancer database analysis. Am Surg 2018;84:1439-1445.

20. Cho IR, Kang H, Jo JH, et al. Efficacy and treatment-related adverse events of gemcitabine plus nab-paclitaxel for treatment of metastatic pancreatic cancer "in a Korean" population: a single-center cohort study. Semin Oncol 2017;44:420427.

21. Versteijne E, Suker M, Groothuis K, et al. Preoperative chemoradiotherapy versus immediate surgery for resectable and borderline resectable pancreatic cancer: results of the Dutch randomized phase III PREOPANC Trial. J Clin Oncol 2020;38:1763-1773.

22. Casadei R, Di Marco M, Ricci C, et al. Neoadjuvant chemoradiotherapy and surgery versus surgery alone in resectable pancreatic cancer: a single-center prospective, randomized, controlled trial which failed to achieve accrual targets. J Gastrointest Surg 2015;19:1802-1812.

23. Fujii T, Satoi S, Yamada S, et al. Clinical benefits of neoadjuvant chemoradiotherapy for adenocarcinoma of the pancreatic head: an observational study using inverse probability of treatment weighting. J Gastroenterol 2017;52:81-93.

24. Ielpo B, Caruso R, Duran H, et al. A comparative study of neoadjuvant treatment with gemcitabine plus nab-paclitaxel versus surgery first for pancreatic adenocarcinoma. Surg Oncol 2017;26:402-410.

25. Suker M, Beumer BR, Sadot E, et al. FOLFIRINOX for locally advanced pancreatic cancer: a systematic review and patient-level meta-analysis. Lancet Oncol 2016;17:801-810.

26. Zhan HX, Xu JW, Wu D, et al. Neoadjuvant therapy in pancreatic cancer: a systematic review and meta-analysis of prospective studies. Cancer Med 2017;6:1201-1219.

27. Golcher H, Brunner TB, Witzigmann H, et al. Neoadjuvant chemoradiation therapy with gemcitabine/cisplatin and surgery versus immediate surgery in resectable pancreatic cancer: results of the first prospective randomized phase II trial. Strahlenther Onkol 2015;191:7-16.

28. Tempero MA. NCCN guidelines updates: pancreatic cancer. J Natl Compr Canc Netw 2019;17:603-605.

29. Cloyd JM, Wang H, Egger ME, et al. Association of clinical factors with a major pathologic response following preoperative therapy for pancreatic ductal adenocarcinoma. JAMA Surg 2017;152:1048-1056.

30. Bissolati M, Sandri MT, Burtulo G, Zorzino L, Balzano G, Braga M. Portal vein-circulating tumor cells predict liver metastases in patients with resectable pancreatic cancer. Tumour Biol 2015;36:991-996.

31. Lee JS, Park SS, Lee YK, Norton JA, Jeffrey SS. Liquid biopsy in pancreatic ductal adenocarcinoma: current status of circulating tumor cells and circulating tumor DNA. Mol Oncol 2019;13:1623-1650. 